\title{
Does the ranking of surgeons in a publicly available online platform correlate with objective outcomes?
}

\author{
Kimon Bekelis, MD, ${ }^{1,2}$ Symeon Missios, MD, ${ }^{3}$ Shannon Coy, MD, ${ }^{4}$ and Jeremiah N. Johnson, MD ${ }^{5}$ \\ ${ }^{1}$ Section of Neurosurgery, Dartmouth-Hitchcock Medical Center; ${ }^{2}$ The Dartmouth Institute for Health Policy and Clinical Practice, \\ Lebanon, New Hampshire; ${ }^{3}$ Department of Neurosurgery, Akron General Hospital, Akron, Ohio; ${ }^{4}$ Department of Pathology, \\ Brigham and Women's Hospital, Boston, Massachusetts; and '5epartment of Neurosurgery, University of Texas Health Science \\ Center, San Antonio, Texas
}

OBJECTIVE The accuracy of public reporting in health care, especially from private vendors, remains an issue of debate. The authors investigated the association of the publicly reported physician complication rates in an online platform with real-world adverse outcomes of the same physicians for patients undergoing posterior lumbar fusion.

METHODS The authors performed a cohort study involving physicians performing posterior lumbar fusions between 2009 and 2013 who were registered in the Statewide Planning and Research Cooperative System database. This cohort was merged with publicly available data over the same time period from ProPublica, a private company. Mixed-effects multivariable regression models were used to investigate the association of publicly available complication rates with the rate of discharge to a rehabilitation facility, length of stay, mortality, and hospitalization charges for the same surgeons.

RESULTS During the selected study period, there were 8,457 patients in New York State who underwent posterior lumbar fusion performed by the 56 surgeons represented in the ProPublica Surgeon Scorecard over the same time period. Using a mixed-effects multivariable regression model, the authors demonstrated that publicly reported physician-level complication rates were not associated with the rate of discharge to a rehabilitation facility (OR $0.97,95 \% \mathrm{Cl} 0.72-1.31$ ), length of stay (adjusted difference $-0.1,95 \% \mathrm{Cl}-0.5$ to 0.2 ), mortality ( $\mathrm{OR} 0.87,95 \% \mathrm{Cl} 0.49-1.55$ ), and hospitalization charges (adjusted difference $\$ 18,735,95 \% \mathrm{Cl}-\$ 59,177$ to $\$ 96,647$ ). Similarly, no association was observed when utilizing propensity score-adjusted models, and when restricting the cohort to a predefined subgroup of Medicare patients.

CONCLUSIONS After merging a comprehensive all-payer posterior lumbar fusion cohort in New York State with data from the ProPublica Surgeon Scorecard over the same time period, the authors observed no association of publicly available physician complication rates with objective outcomes.

https://thejns.org/doi/abs/10.3171/2016.8.JNS16583

KEY WORDS ProPublica Surgeon Scorecard; posterior lumbar fusion; public reporting; outcomes; SPARCS;

Statewide Planning and Research Cooperative System

$\mathrm{A}$ $s$ the practice of medicine increasingly focuses on accountability, the quality of surgical interventions is under scrutiny by patients, peers, payers, and policy makers.,7 Quality measurement and public reporting have a central role in this new and evolving health care landscape. ${ }^{4}$ The recently passed ${ }^{18}$ MACRA (Medicare Access and CHIP Reauthorization Act) highlights the development of several public reporting avenues for quality metrics. The CMS (Centers for Medicare and Medicaid Services) has been a pioneer in this area by establishing the first reporting platform through the Hospital
Compare and Physician Compare websites (https://www. medicare.gov/hospitalcompare; https://www.medicare.gov/ physiciancompare). Several private vendors are also starting to launch similar online platforms. The common goal of all of these initiatives is to increase patient awareness about physicians and hospitals before selecting where they will undergo procedures or receive care.

Although if done appropriately and such benchmarking can empower all participants in the health care debate, $, 4,28$ concerns have been raised about the appropriateness and validity of currently reported measures, especially for not

ABBREVIATIONS LOS $=$ length of stay; SPARCS $=$ Statewide Planning and Research Cooperative System.

SUBMITTED March 7, 2016. ACCEPTED August 5, 2016.

INCLUDE WHEN CITING Published online November 11, 2016; DOI: 10.3171/2016.8.JNS16583. 
publicly accountable private vendors. ${ }^{8}, 15$ Critics of such ranking systems ${ }^{14}$ point out the potential for misleading information given unclear methodology and the limited consideration of important objective metrics. ${ }^{1}$ In addition, the lack of public oversight has fueled a debate on the appropriateness of the reporting of such indices by privately held companies. ${ }^{15}$ There has been no prior study attempting to validate these claims in a comprehensive way for a private vendor.

We used the New York Statewide Planning and Research Cooperative System (SPARCS; http://www.health. ny.gov/statistics/sparcs/) database to study the association of the ranking of surgeons based on complication rates reported by ProPublica, a private company publicly reporting quality measures, with objective outcomes (discharge to a rehabilitation facility, length of stay [LOS], mortality, and hospitalization charges) of the same surgeons calculated in SPARCS for patients undergoing posterior lumbar fusion. Several multivariable regression models were used to control for confounding. These included a propensity score-adjusted regression model and a mixed-effects model to control for clustering at the physician level.

\section{Methods \\ New York SPARCS}

All patients undergoing posterior lumbar fusion procedures who were registered in the SPARCS (New York State Department of Health, Albany, NY) database between 2009 and 2013 were included in the analysis. For these years, SPARCS contains patient-level details for every hospital discharge, ambulatory surgery, and emergency department admission in New York State as coded from admission and billing records. More information about SPARCS is available at https://www.health.ny.gov/ statistics/sparcs/.

\section{ProPublica Surgeon Scorecard}

The ProPublica Surgeon Scorecard is a public reporting program developed by ProPublica, a private news-reporting company, in collaboration with a paid biostatistician consultant from Harvard School of Public Health. It reports risk-adjusted complication rates for 8 common elective surgical procedures based only on Medicare data between 2009 and 2013. Complications are defined as inpatient mortality or 30-day readmissions related to the operation as determined by a panel of experts. Mixed-effects models were used for risk adjustment. Rates are reported only for physicians performing more than 20 procedures for patients covered by Medicare. The website is designed in such a way that patients can make side-by-side comparisons of physicians who are geographically close to one another. More information is available at https://projects. propublica.org/surgeons/.

\section{Cohort Definition}

To establish the cohort of patients, we used ICD-9$\mathrm{CM}$ codes to identify patients in SPARCS who underwent posterior lumbar fusions (ICD-9-CM codes 81.05, 81.07, 81.08, 81.35, and 81.38) between 2009 and 2013. We merged SPARCS and ProPublica data over the same time periods using the physician New York license numbers. All physicians represented in ProPublica were matched to their records in SPARCS for the corresponding years.

\section{Outcome Variables}

The primary outcome variable was discharge to a rehabilitation facility after posterior lumbar fusion. This was defined as discharge to any facility other than the patient's home. Secondary outcomes were LOS, mortality, and total hospitalization charges during the initial hospitalization.

These outcomes were selected as the only available outcomes through administrative data (similar to what ProPublica is using) that are to some degree directly or indirectly associated with the operating surgeon.

\section{Exposure Variables}

The primary exposure variable was the surgeon's riskadjusted complication rate, as publicly reported in the ProPublica Surgeon Scorecard. This was treated as a continuous variable. For part of the analysis (propensity score adjustment), complication rate was treated as a binary variable. The cutoff to determine whether the surgeon had a high complication rate was the median complication rate of all surgeons in our data.

Covariates (Supplemental Table 1) used for risk adjustment included age, sex, race (African American, Hispanic, Asian, Caucasian, other), and insurance (private, Medicare, Medicaid, uninsured, other). The comorbidities used for risk adjustment were diabetes mellitus, smoking, chronic lung disease, hypertension, hypercholesterolemia, peripheral vascular disease, congestive heart failure, coronary artery disease, history of ischemic stroke, history of transient ischemic attack, alcohol abuse, obesity, chronic renal failure, and coagulopathy. Only variables that were defined as "present on admission" were considered as part of the patient's preadmission comorbidity profile.

\section{Statistical Analysis}

The association of ProPublica complication rates with our outcome measures was examined in a multivariable setting. A logistic regression was employed for categorical outcomes (mortality and discharge to a facility) and a linear regression for linear outcomes (LOS and hospitalization charges). The linear models were repeated after logarithmic transformation of the outcomes. The direction of the observed associations did not change, and therefore these results are not reported further for ease of interpretation. The covariates used for risk adjustment in our models were age, sex, race, insurance, and all the comorbidities mentioned previously. Mixed-effects methods were employed, with operating surgeon used as a random-effects variable, to account for clustering at the surgeon level.

In an alternative method to control for confounding, we used a propensity-adjusted (with deciles of propensity score) regression model. We calculated the propensity of undergoing treatment by a "high complication" surgeon with a separate logistic regression model, using all the covariates mentioned previously. Propensity adjustment compares subjects within the same decile of propensity score, balancing potential differences in covariates, while 
TABLE 1. Patient characteristics

\begin{tabular}{|c|c|c|c|}
\hline Characteristic & $\begin{array}{c}\text { All } \\
\text { Patients }\end{array}$ & $\begin{array}{l}\text { Patients Treated by Physicians } \\
\text { w/ Low Complication Rates }\end{array}$ & $\begin{array}{l}\text { Patients Treated by Physicians } \\
\text { w/ High Complication Rates* }\end{array}$ \\
\hline Sample size & 8,457 & 4,151 & 4,306 \\
\hline Mean age, yrs (SD) & $58.7(14.8)$ & $59.5(14.8)$ & $58.1(14.7)$ \\
\hline Female sex & $4,508(53.3 \%)$ & $2,279(54.9 \%)$ & $2,231(51.8 \%)$ \\
\hline \multicolumn{4}{|l|}{ Insurance status } \\
\hline Medicare & $2,958(35.0 \%)$ & $1,551(37.4 \%)$ & $1,411(32.8 \%)$ \\
\hline Medicaid & $220(2.6 \%)$ & $91(2.2 \%)$ & $125(2.9 \%)$ \\
\hline Private insurance & $3,938(46.6 \%)$ & $1,779(42.9 \%)$ & $2,156(50.1 \%)$ \\
\hline Self-pay & $237(2.8 \%)$ & $199(4.8 \%)$ & $39(0.9 \%)$ \\
\hline Other & $1,107(13.1 \%)$ & $531(12.8 \%)$ & $572(13.3 \%)$ \\
\hline \multicolumn{4}{|l|}{ Race } \\
\hline Caucasian & $7,171(85.3 \%)$ & $3,388(81.9 \%)$ & $3,783(88.6 \%)$ \\
\hline African American & $429(5.1 \%)$ & $248(6.0 \%)$ & $184(4.3 \%)$ \\
\hline Hispanic & $244(2.9 \%)$ & $128(3.1 \%)$ & $111(2.6 \%)$ \\
\hline Asian & $101(1.2 \%)$ & $66(1.6 \%)$ & $34(0.8 \%)$ \\
\hline Other & $462(5.5 \%)$ & $306(7.4 \%)$ & $154(3.6 \%)$ \\
\hline \multicolumn{4}{|l|}{ Comorbidities } \\
\hline Diabetes mellitus & $1,311(15.5 \%)$ & $627(15.1 \%)$ & $685(15.9 \%)$ \\
\hline Tobacco use & $1,252(14.8 \%)$ & $648(15.6 \%)$ & $603(14.0 \%)$ \\
\hline Obesity & $1,125(13.3 \%)$ & $498(12.0 \%)$ & $624(14.5 \%)$ \\
\hline History of ischemic stroke & $132(0.01 \%)$ & $72(0.02 \%)$ & $0(0 \%)$ \\
\hline CAD & $1,235(11.4 \%)$ & $486(11.7 \%)$ & $478(11.1 \%)$ \\
\hline COPD & $1,235(14.6 \%)$ & $614(14.8 \%)$ & $620(14.4 \%)$ \\
\hline $\mathrm{CHF}$ & $186(2.2 \%)$ & $104(2.5 \%)$ & $86(2.0 \%)$ \\
\hline Coagulopathy & $118(1.4 \%)$ & $58(1.4 \%)$ & $60(1.4 \%)$ \\
\hline CRF & $237(2.8 \%)$ & $125(3.0 \%)$ & $112(2.6 \%)$ \\
\hline Hypertension & $4,237(50.1 \%)$ & $2,117(51.0 \%)$ & $4,775(49.2 \%)$ \\
\hline Hypercholesterolemia & $3,028(35.8 \%)$ & $1,482(35.7 \%)$ & $2,119(35.9 \%)$ \\
\hline Alcohol abuse & $118(1.4 \%)$ & $71(1.7 \%)$ & $43(1.0 \%)$ \\
\hline PVD & $220(2.6 \%)$ & $95(2.3 \%)$ & $125(2.9 \%)$ \\
\hline
\end{tabular}

$\mathrm{CAD}=$ coronary artery disease; $\mathrm{CHF}$ = congestive heart failure; $\mathrm{COPD}=$ chronic obstructive pulmonary disease; $\mathrm{CRF}$ = chronic renal failure; $P V D=$ peripheral vascular disease.

* Greater than the average complication rates.

using the entire cohort, without any observations being discarded. Mixed-effects methods were employed, with operating surgeon used as a random-effects variable, to account for clustering at the surgeon level.

As part of a sensitivity analysis, we restricted our cohort to just the 2 ICD-9-CM codes used by ProPublica to define posterior lumbar fusion $(81.07,81.08)$. In addition, we restricted our analysis to only Medicare patients, which is the subgroup the original surgeon's scorecard was built on.

For the calculation of risk-adjusted rates of outcomes, we employed a mixed-effects predictive model including age, sex, race, insurance, and all the comorbidities mentioned previously. Hospital of treatment was used as a random-effects variable.

Regression diagnostics were used for all models. All results are based on 2-sided tests, and the level of statistical significance was set at 0.05 . Only cases without missing values were included in the analysis. This study, based on 8,457 patients, has sufficient power $(80 \%)$ at a $5 \%$ Type I error rate to detect differences in discharge to a facility, as small as 3\%. Statistical analyses were performed using the 64-bit version of R.3.1.0 (R Foundation for Statistical Computing), Stata (version 13, StataCorp.), and SPSS (version 22, IBM).

\section{Results}

\section{Patient Characteristics}

During the selected study period there were $8,457 \mathrm{pa}$ tients in New York State who underwent posterior lumbar fusion (mean age 58.7 years, 53.3\% of patients were female) performed by 56 surgeons who were also represented in the ProPublica Surgeon Scorecard. During the same period, 2,897 patients (25.5\%) underwent posterior lumbar fusion operations by surgeons not represented in 
TABLE 2. Association of comprehensive surgeon outcomes with surgeon complication rates as reported in ProPublica Surgeon Scorecard

\begin{tabular}{|c|c|c|c|c|c|c|c|c|}
\hline \multirow[b]{2}{*}{ Model } & \multicolumn{2}{|c|}{ Discharge to Rehabilitation* } & \multicolumn{2}{|l|}{ LOS (days) $\dagger$} & \multicolumn{2}{|c|}{ Inpatient Mortality* } & \multicolumn{2}{|l|}{ Hospitalization Charges† } \\
\hline & $\begin{array}{c}\text { OR } \\
(95 \% \mathrm{Cl})\end{array}$ & $\begin{array}{c}p \\
\text { Value }\end{array}$ & $\begin{array}{c}\text { Adjusted Difference } \\
\qquad(95 \% \mathrm{Cl})\end{array}$ & $\begin{array}{c}p \\
\text { Value }\end{array}$ & $\begin{array}{c}\text { OR } \\
(95 \% \mathrm{Cl})\end{array}$ & $\begin{array}{c}p \\
\text { Value }\end{array}$ & $\begin{array}{c}\text { Adjusted Difference } \\
\qquad(95 \% \mathrm{Cl})\end{array}$ & $\begin{array}{c}p \\
\text { Value }\end{array}$ \\
\hline Crude & $0.90(0.86-0.93)$ & $<0.001$ & $-0.2(-0.3$ to -0.1$)$ & $<0.001$ & $0.83(0.53-1.30)$ & 0.422 & $\$ 15,805$ ( $\$ 2,985$ to $\$ 28,624)$ & $<0.001$ \\
\hline $\begin{array}{l}\text { Multivariable } \\
\text { regression } \ddagger\end{array}$ & $0.97(0.72-1.31)$ & 0.834 & $-0.1(-0.5$ to 0.2$)$ & 0.428 & $0.87(0.49-1.55)$ & 0.646 & $\$ 18,735(-\$ 59,177$ to $\$ 96,647)$ & 0.637 \\
\hline $\begin{array}{c}\text { Propensity score } \\
\text { adjustment } \neq\end{array}$ & $0.65(0.34-1.24)$ & 0.196 & $-0.2(-1.0$ to 0.7$)$ & 0.659 & $0.86(0.37-2.00)$ & 0.723 & $\$ 48,946(-\$ 22,721$ to $\$ 12,932)$ & 0.590 \\
\hline
\end{tabular}

Propensity score calculations were based on age, sex, race, insurance, and all comorbidities. Complication rates were treated as a binary variable (with rates higher than average considered "high") for the purposes of regression adjustment.

* Analyses based on logistic regression.

$\dagger$ Analyses are based on linear regression.

$\ddagger$ Mixed effects; includes surgeon as a random-effects variable.

the ProPublica Surgeon Scorecard. The characteristics of our cohort at baseline can be seen in Table 1.

\section{Discharge to a Facility}

Overall, 3,873 patients $(45.8 \%)$ were discharged to a facility after posterior lumbar fusion in our cohort. Higher publicly reported physician complication rates were associated with a lower rate of discharge to a facility (OR 0.90, 95\% CI 0.86-0.93) in the unadjusted analysis. However, after adjusting for confounding in a mixed-effects multivariable regression model (Table 2), ProPublica complication rates were not associated with discharge to a rehabilitation facility (OR 0.97, 95\% CI 0.72-1.31). This persisted in a propensity score-adjusted model (OR $0.65,95 \%$ CI $0.34-1.24)$. Figure 1A demonstrates the correlation $(\mathrm{r}=$ $-0.0009)$ of risk-adjusted rates of discharge to a rehabilitation facility with the risk-adjusted ProPublica complication rates for surgeons in our study.

\section{LOS}

The average LOS was 5.1 days (SD 5.6) after posterior lumbar fusion in our cohort. Higher publicly reported physician complication rates were associated with shorter LOS (difference $-0.2,95 \% \mathrm{CI}-0.3$ to -0.1 ) in the unadjusted analysis. However, after adjusting for confounding in a mixed-effects multivariable regression model (Table 2), ProPublica complication rates were not associated with LOS (adjusted difference $-0.1,95 \% \mathrm{CI}-0.5$ to 0.2 ). This persisted in a propensity score-adjusted model (adjusted difference $-0.2,95 \% \mathrm{CI}-1.0$ to 0.7 ). Figure $1 \mathrm{~B}$ demonstrates the correlation $(r=-0.009)$ of risk-adjusted LOS with the risk-adjusted ProPublica complication rates for surgeons in our study.

\section{Inpatient Mortality}

Overall, $17(0.2 \%)$ inpatient deaths were recorded after posterior lumbar fusion in our cohort. Publicly reported physician complication rates were not associated with mortality (OR $0.83,95 \%$ CI $0.53-1.30$ ) in the unadjusted analysis. This persisted (Table 2) in a mixed-effects logistic regression model (OR 0.87, 95\% CI 0.49-1.55) and a propensity score-adjusted model (OR $0.86,95 \%$ CI
0.37-2.00). Figure 1C demonstrates the correlation ( $\mathrm{r}=$ -0.018 ) of risk-adjusted mortality with the risk-adjusted ProPublica complication rates for surgeons in our study.

\section{Hospitalization Charges}

The average hospitalization charges were $\$ 82,504$ (SD $\$ 65,971$ ) after posterior lumbar fusion in our cohort. Higher publicly reported physician complication rates were associated with higher total hospitalization charges (difference $\$ 15,805,95 \%$ CI $\$ 2,985-\$ 28,624$ ) in the unadjusted analysis. However, after adjusting for confounding in a mixed-effects multivariable regression model (Table 2), ProPublica complication rates were not associated with total hospitalization charges (adjusted difference $\$ 18,735$, $95 \%$ CI $-\$ 59,177$ to $\$ 96,647$ ). This persisted in a propensity score-adjusted model (adjusted difference $\$ 48,946$, $95 \%$ CI $-\$ 22,721$ to $\$ 12,932)$. Figure 1D demonstrates the correlation $(\mathrm{r}=0.02)$ of risk-adjusted hospitalization charges with the risk-adjusted ProPublica complication rates for surgeons in our study.

\section{Sensitivity Analysis}

In sensitivity analysis, restricting our cohort to the ICD-9-CM codes used by ProPublica, higher publicly reported physician complication rates were not associated with rates of discharge to a facility (OR 0.94, 95\% CI $0.69-1.28$ ), mortality (OR $1.44,95 \%$ CI $0.42-4.94$ ), LOS (adjusted difference $-0.03,95 \%$ CI -0.29 to 0.23 ), and total hospitalization charges (adjusted difference $\$ 10,763,95 \%$ CI $-\$ 12,207$ to $\$ 22,749)$. When restricting the cohort to just Medicare patients, we did not identify an association of publicly reported physician complication rates with rates of discharge to a facility (OR $0.81,95 \% \mathrm{CI}$ $0.54-1.22$ ), mortality (OR $0.90,95 \%$ CI $0.86-0.93$ ), LOS (adjusted difference $-0.04,95 \% \mathrm{CI}-0.4$ to 0.5 ), and total hospitalization charges (adjusted difference \$7,245, 95\% CI $-\$ 9,846$ to $\$ 24,336)$.

\section{Discussion}

Using a comprehensive all-payer cohort of patients in New York State undergoing posterior lumbar fusion, we identified no association of publicly reported complication 

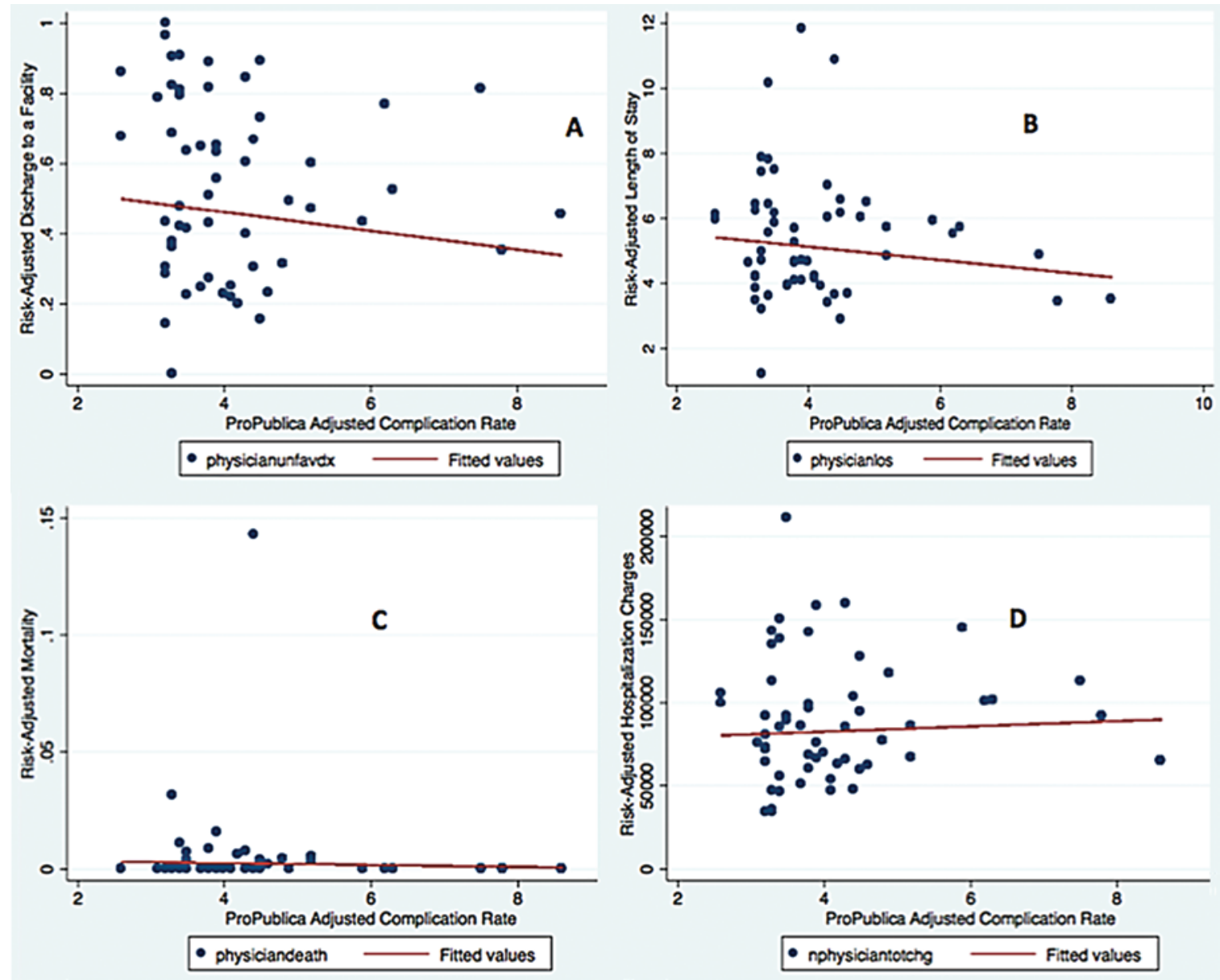

FIG. 1. Scatterplots demonstrating the correlation of risk-adjusted rates of surgeon complications as reported in the ProPublica Surgeon Scorecard and the risk-adjusted rates of discharge to a facility $(\mathbf{A})$, length of stay (B), mortality (C), and total hospitalization charges (D) for the same surgeons in New York State over the same period of time. Figure is available in color online only.

rates by a private vendor with the rate of discharge to rehabilitation, LOS, mortality, and hospitalization charges for the same surgeons calculated in the database over the same time period. Our results were robust when considering several advanced observational techniques to account for confounders. The science of quality measurement will be central in guiding patient choices. ${ }^{7}$ Reliable public reporting is expected to raise quality of care, lower average cost, and improve population health. ${ }^{11,14,28}$ ProPublica is one of the first private endeavors to report surgeon complications, and it complements similar efforts by the government. ${ }^{23}$ However, there are concerns that the methodology employed and the metrics reported might be misleading for patient choices.

Prior observational studies have reported conflicting results on the validity of publicly reported quality measures by government agencies. Werner and Bradlow ${ }^{29}$ demonstrated that disease-specific Hospital Compare metrics for pneumonia, myocardial infarction, and congestive heart failure were associated with modestly improved mortality. In addition, Stulberg et al., ${ }^{27}$ using a commercial database, identified an association of process measure performance and decreased rates of surgical site infections. In contrast,
Shafi et al., ${ }^{25}$ in a study of the National Trauma Data Bank, demonstrated that the performance of trauma centers on Hospital Compare metrics was not associated with outcomes of trauma patients. In their conclusions, they advocated for the development of trauma-specific measures. More recently, other investigators ${ }^{20}$ were not able to identify an association of subjective Hospital Compare metrics with objective outcomes of patients undergoing spinal surgery in New York State. In other studies, Safavi et al. ${ }^{24}$ and Paddock et al..$^{21}$ concluded that publicly reported measures make it difficult to distinguish among hospitals from which patients are likely to choose. No prior investigation has focused on reported quality measures by nonpublicly accountable private organizations.

The present analysis demonstrated no association of objective outcomes with complication rates reported in ProPublica. There are several methodological drawbacks ${ }^{10}$ in the creation of the surgeon scorecard that might explain our findings. First, the ProPublica is based on fee-forservice Medicare claims. This represents $20 \%$ of all insurance coverage and excludes privately insured patients, Medicaid, managed care, and other insurance products. Physician practices vary widely in regard to their payer 
mix, which introduces a significant selection bias. Most notably, some of the highest volume surgeons are not represented in ProPublica because they do not reach the cutoff of 20 Medicare procedures arbitrarily set by the authors. Second, the ICD-9-CM codes employed by ProPublica to define the spine surgery cohort are inaccurate and misrepresent the procedures performed. Coding is very diverse in different institutions, and there is no single code used by all providers to represent a spinal fusion. Prior peer-reviewed studies of lumbar fusion and spinal surgery in general ${ }^{2,5,13,16,19}$ have used multiple codes in their definitions, a methodology which we have also followed in our study. However, even when restricting our cohort to the limited codes used by ProPublica our results remain. Third, the definition of complications based on administrative data does not represent the granularity needed for patient choices. Such quality improvement efforts are currently underway, spearheaded by specialty societies..$^{17}$ In addition, selecting complications focusing on readmissions and expert opinion ${ }^{22}$ ignores the fact that the majority of complications happen during the patient's hospitalization, resulting in death or unfavorable discharge. Readmissions are, to a large degree, irrelevant to surgeon skill and are dependent on continuity of care and the systems put in place by hospitals to facilitate the postdischarge needs of the patients. ${ }^{3,6}$ Lastly, ProPublica does not provide a breakdown of the individual metrics comprising its complication measure and offers no information on missing data.

From a policy perspective, we found no evidence that patients should base their decisions on reported complication rates by ProPublica. Such metrics do not currently allow the side-by-side comparison of different physicians in regard to the quality of care in spine surgery. In order for this goal to be met, specialty-specific, patientcentered, quality-oriented outcomes should be monitored and reported. Recent legislative reform ${ }^{18}$ allows a more active role for registries in public reporting, avoiding the long and costly task of developing metrics through the National Quality Forum (http://www.qualityforum.org/Measuring Performance/Consensus_Development_Process.aspx). In this direction, the NeuroPoint Alliance (http://www. neuropoint.org) is creating the first neurosurgery-specific Qualified Clinical Data Registry Reporting, based on metrics developed as part of the Quality and Outcomes Database (formerly the $\mathrm{N}^{2} \mathrm{QOD}$ [National Neurosurgical Quality and Outcomes Database]). ${ }^{17}$ Similar initiatives are underway by general surgery through the National Surgical Quality Initiative Program (https://www.facs.org/ quality-programs/acs-nsqip). These specific measures, in the setting of rigorous risk adjustment, will allow the headto-head comparison of physicians.

Quality measurement and reporting is an immature science. ${ }^{14,30}$ However, a recent survey-based study demonstrated a strong influence of quality metrics on the decisions of hospital leaders, despite their concerns about the clinical meaningfulness, unintended consequences, and methods of public reporting. ${ }^{12,15}$ Currently, different quality initiatives use various metrics to create ratings, some of which are based on numerical data and some on solicited opinion. Methodologies are widely varied and results are presented in different ways. ${ }^{30}$ Most importantly, rating systems often disagree with each other, ${ }^{1}$ highlighting the complexity and multidimensionality of the enterprise. ${ }^{9,26}$ The aforementioned specialty society initiatives aim to create universal metrics of quality to allow patient participation in the selection process.

Our study has several limitations common to administrative databases. Residual confounding will undoubtedly be present. However, we used advanced observational techniques to control for confounders. In addition, coding inaccuracies can affect our estimates. However, we used codes that have been employed by prior studies of spinal surgery ${ }^{2,5}$ and are more representative than the codes used by ProPublica. Although SPARCS includes all hospitals from the entire New York State, the generalization of this analysis to the US population at large is uncertain. Additionally, we were lacking post-hospitalization and longterm data on our patients. Quality metrics (i.e., modified Rankin Scale score) are also not available through this database, and therefore we cannot compare our patients on these outcomes. Initiatives, such as the Quality and Outcomes Database, will provide such opportunities in the future. These clinical measures will be crucial to compare surgeons on procedure-specific metrics. Finally, causality cannot be definitively established based on observational data, despite the use of advanced techniques.

\section{Conclusions}

The accuracy of public reporting in health care, especially from private vendors, remains an issue of debate. Using a comprehensive all-payer cohort of patients in New York State undergoing posterior lumbar fusions, we identified no association of publicly reported complication rates by a private vendor with the rate of discharge to rehabilitation, LOS, mortality, and hospitalization charges for the same surgeons calculated in the database over the same time period.

\section{Acknowledgments}

This study was supported by a grant from the National Center for Advancing Translational Sciences (NCATS) of the NIH (Dartmouth Clinical and Translational Science Institute-UL1TR001086). The funders had no role in the design or execution of the study.

\section{References}

1. Austin JM, Jha AK, Romano PS, Singer SJ, Vogus TJ, Wachter RM, et al: National hospital ratings systems share few common scores and may generate confusion instead of clarity. Health Aff (Millwood) 34:423-430, 2015

2. Bekelis K, Desai A, Bakhoum SF, Missios S: A predictive model of complications after spine surgery: the National Surgical Quality Improvement Program (NSQIP) 2005-2010. Spine J 14:1247-1255, 2014

3. Bekelis K, Fisher ES, Labropoulos N, Zhou W, Skinner J: Variations in the intensive use of head CT for elderly patients with hemorrhagic stroke. Radiology 275:188-195, 2015

4. Bekelis K, Goodney RP, Dzebisashvili N, Goodman DC, Bronner KK: Variation in the care of surgical conditions: cerebral aneurysms, in Goodney PR, Dzebisashvili N, Goodman DC (eds): Variation in the Care of Surgical Conditions. A Dartmouth Atlas of Health Care Series. Lebanon, NH: Dartmouth Institute, 2015

5. Bekelis K, Missios S: The association of regional intensity 
of neurosurgical care with spinal fusion surgery in the USA. Eur Spine J 23:909-915, 2014

6. Bekelis K, Roberts DW, Zhou W, Skinner JS: Fragmentation of care and the use of head computed tomography in patients with ischemic stroke. Circ Cardiovasc Qual Outcomes 7:430-436, 2014

7. Birkmeyer NJ, Birkmeyer JD: Strategies for improving surgical quality - should payers reward excellence or effort? $\mathbf{N}$ Engl J Med 354:864-870, 2006

8. Cassel CK, Conway PH, Delbanco SF, Jha AK, Saunders RS, Lee TH: Getting more performance from performance measurement. N Engl J Med 371:2145-2147, 2014

9. Donabedian A: Evaluating the quality of medical care. 1966. Milbank Q 83:691-729, 2005

10. Friedberg MW, Pronovost PJ, Shahian DM, Safran DG, Bilimoria KY, Elliott MN, et al: A Methodological Critique of the ProPublica Surgeon Scorecard. Santa Monica, CA: RAND Corporation, 2015 (http://www.rand.org/content/dam/ rand/pubs/perspectives/PE100/PE170/RAND_PE170.pdf) [Accessed September 15, 2016]

11. Fung CH, Lim YW, Mattke S, Damberg C, Shekelle PG: Systematic review: the evidence that publishing patient care performance data improves quality of care. Ann Intern Med 148:111-123, 2008

12. Goff SL, Lagu T, Pekow PS, Hannon NS, Hinchey KL, Jackowitz TA, et al: A qualitative analysis of hospital leaders' opinions about publicly reported measures of health care quality. Jt Comm J Qual Patient Saf 41:169-176, 2015

13. Gray DT, Deyo RA, Kreuter W, Mirza SK, Heagerty PJ, Comstock BA, et al: Population-based trends in volumes and rates of ambulatory lumbar spine surgery. Spine (Phila Pa 1976) 31:1957-1964, 2006

14. Harder B, Comarow A: Hospital quality reporting by US News \& World Report: why, how, and what's ahead. JAMA 313:1903-1904, 2015

15. Lindenauer PK, Lagu T, Ross JS, Pekow PS, Shatz A, Hannon N, et al: Attitudes of hospital leaders toward publicly reported measures of health care quality. JAMA Intern Med 174:1904-1911, 2014

16. Martin BI, Deyo RA, Mirza SK, Turner JA, Comstock BA, Hollingworth W, et al: Expenditures and health status among adults with back and neck problems. JAMA 299:656-664, 2008

17. McGirt MJ, Speroff T, Dittus RS, Harrell FEJ Jr, Asher AL: The National Neurosurgery Quality and Outcomes Database $\left(\mathrm{N}^{2} \mathrm{QOD}\right)$ : general overview and pilot-year project description. Neurosurg Focus 34(1):E6, 2013

18. Medicare Access and CHIP Reauthorization Act of 2015, Pub L 114-10

19. Missios S, Bekelis K: Hospitalization cost after spine surgery in the United States of America. J Clin Neurosci 22:16321637,2015

20. Missios S, Bekelis K: How well do subjective Hospital Compare metrics reflect objective outcomes in spine surgery? J Neurosurg Spine 25:264-270, 2016

21. Paddock SM, Adams JL, Hoces de la Guardia F: Better-thanaverage and worse-than-average hospitals may not significantly differ from average hospitals: an analysis of Medicare Hospital Compare ratings. BMJ Qual Saf 24:128-134, 2015
22. Pierce O, Allen M: Assessing Surgeon-Level Risk of Patient Harm During Elective Surgery for Public Reporting. New York: ProPublica, 2015

23. Ryan AM, Nallamothu BK, Dimick JB: Medicare's public reporting initiative on hospital quality had modest or no impact on mortality from three key conditions. Health Aff (Millwood) 31:585-592, 2012

24. Safavi KC, Dai F, Gilbertsen TA, Schonberger RB: Variation in surgical quality measure adherence within hospital referral regions: do publicly reported surgical quality measures distinguish among hospitals that patients are likely to compare? Health Serv Res 49:1108-1120, 2014

25. Shafi S, Parks J, Ahn C, Gentilello LM, Nathens AB, Hemmila MR, et al: Centers for Medicare and Medicaid services quality indicators do not correlate with risk-adjusted mortality at trauma centers. J Trauma 68:771-777, 2010

26. Sheps MC: Approaches to the quality of hospital care. Public Health Rep 70:877-886, 1955

27. Stulberg JJ, Delaney CP, Neuhauser DV, Aron DC, Fu P, Koroukian SM: Adherence to surgical care improvement project measures and the association with postoperative infections. JAMA 303:2479-2485, 2010

28. Werner RM, Bradlow ET: Public reporting on hospital process improvements is linked to better patient outcomes. Health Aff (Millwood) 29:1319-1324, 2010

29. Werner RM, Bradlow ET: Relationship between Medicare's hospital compare performance measures and mortality rates. JAMA 296:2694-2702, 2006

30. Zuger A: Hospital ratings: a guide for the perplexed. JAMA 313:1911-1912, 2015

\section{Disclosures}

The authors report no conflict of interest concerning the materials or methods used in this study or the findings specified in this paper.

\section{Author Contributions}

Conception and design: Bekelis, Johnson. Acquisition of data: Missios, Coy, Johnson. Analysis and interpretation of data: Bekelis, Missios, Coy. Drafting the article: Bekelis. Critically revising the article: Missios, Coy, Johnson. Reviewed submitted version of manuscript: Bekelis. Approved the final version of the manuscript on behalf of all authors: Bekelis. Statistical analysis: Bekelis. Study supervision: Bekelis.

\section{Supplemental Information Online-Only Content}

Supplemental material is available with the online version of the article.

Supplemental Table 1. https://thejns.org/doi/suppl/10.3171/ 2016.8.JNS16583.

\section{Correspondence}

Kimon Bekelis, Section of Neurosurgery, Dartmouth-Hitchcock Medical Center, One Medical Center Dr., Lebanon, NH 03756. email: kbekelis@gmail.com. 